\title{
EL SISTEMA ESCUELA: UN ACERCAMIENTO DESDE EL PARADIGMA DE LA COMPLEJIDAD
}

\author{
Aquiles Alejandro Almonacid Fierro
}

"El futuro es un campo de batalla/ de honor o de trigales que está quieto o que viene timorato/ una vez será julio de nevadas otra vez será enero de canículas...

El futuro será/ qué duda cabe/ una puerta/ la única que no sabe cerrarse... (Mario Benedetti)

\section{RESUMEN}

El presente documento, pretende entregar una mirada acerca del sistema escolar, a partir de las nociones de complejidad, enfoque sistémico y concepción epistemológica, que permita reflexionar sobre los procesos educativos que desarrolla la escuela en procura de la denominada "calidad educativa". El planteamiento es que de continuar las existentes condiciones, vale decir, aplicando modelos lineales y sumativos fuertemente arraigados en el paradigma de la simplicidad, es muy difícil responder a las expectativas que la sociedad ha depositado en la sistema educativo, puesto que en la actualidad la navegación por el océano del conocimiento requiere nuevas coordenadas, nuevas cartas de navegación, nuevos instrumentos, para emigrar del archipiélago al continente.

Palabras claves: escuela - sistema - complejidad - epistemología.

\section{SCHOOL SYSTEM: AN APPROACH FROM THE PARADIGM OF COMPLEXITY}

\begin{abstract}
This article aims to give a look about the school system, from the notions of complexity, systemic and epistemological approach that allows reflection on educational processes developed by the school in pursuit of the so-called "quality education". The approach is to continue the existing conditions, i.e., using linear models and summative deeply rooted in the paradigm of simplicity, it is very difficult to respond to the expectations that society has in the education system, since currently the navigating by the ocean of knowledge requires new dimensions, new charts, new instruments, to emigrate from the islands to the mainland.
\end{abstract}

Key Words: school - system- complexity- epistemology

* Profesor de Educación Física, Magíster en Motricidad Humana. Docente Universidad Autónoma de Chile sede Talca.E mail: aalmonacidf@uautonoma.cl 


\section{Primeras Navegaciones}

Desde pequeño imaginaba el siglo XXI como un tiempo repleto de robots que realizarían las más variadas funciones y tareas, desde el aseo de una casa, el cuidado del jardín, conducir un vehículo, hasta dar una clase en la escuela, la comida se compondría de pequeñas cápsulas de colores que suplirían todas mis necesidades energéticas -qué pena por el mantecado, mi helado favorito de la infancia-; en ese futuro lejano me podría trasladar de un sitio a otro volando en un vehículo especialmente diseñado para mí, ya no habrían casas, sólo edificios, edificios por todos lados que permitirían albergar a mucha gente trabajando en minúsculas oficinas con pantallas y papeles por doquier.

El año 2000 se veía remoto e incierto, tanto así, que en muchas oportunidades me pregunté si alcanzaría a vivir en aquella época, para poder disfrutar de la maravilla de los botones, capsulas, autos voladores y de los ingenuos pero eficientes robots, que se convertirían en tan buenos amigos como mi querido Sultán, el perro fiel que me acompañó con sus miradas y sonrisas hasta entrada la adolescencia, para luego irse de un día para otro, sin siquiera despedirse.

Hoy, en los albores del siglo XXI, puedo evocar el pasado con la nostalgia de aquella infancia desvanecida por el paso del tiempo, y ver con la mirada de un adulto cómo la mayoría de aquellas escenas que me quitaban el sueño, son todavía "el futuro". Por otro lado, hay cosas que poco han cambiado con el paso de los años, como la noble escuela que me albergó durante los primeros ocho años de mi vida escolar, con sus salas rectangulares, su pizarra negra, sus sillas y bancos de madera, sus profesores, mis queridos maestros, la mayoría ya jubilados -otros fallecidos- luego de enseñar a una generación tras otra a leer, sumar, escribir, dividir, cantar, dibujar...; mi escuela continúa en el mismo lugar del que la abandoné hace casi 30 años y salvo pequeñas transformaciones, es idéntica a la que se encuentra albergada en mis recuerdos.

Y es precisamente esta escuela -más específicamente la escuela del tercer milenio-la que hoy ocupa mis desvelos, el sueño se interrumpe, llegan 
las imágenes y las preguntas golpean mi ventana, para luego de abrirla escurrirse delicadamente por mi almohada y acompañarme noche tras noche, con su inquietante zumbido de conversaciones y risas.

\section{2. ¿Un nuevo paradigma para re-mirar la escuela?}

¿Qué es la escuela? La pregunta que en un principio parece simple nos aturde, nos desarma, nos desnuda. Desde el paradigma clásico, vale decir, aquel que se caracteriza por la linealidad, la proporcionalidad, la certidumbre, el empirismo, y sobre todo, la disyunción cartesiana, base del método analítico (Boisier, 2003), la respuesta es casi evidente: la escuela es el lugar donde se desarrolla el acto educativo, la formación de personas y la transmisión de la cultura. Podríamos agregar que posee una estructura compuesta por elementos tales como infraestructura -generalmente un edificio-, materiales o insumos, alumnos, profesores, apoderados, planes de estudio, en fin, podríamos entregar una larga lista de los elementos que constituyen y dan forma a la escuela, en una actitud más bien sumativa, de abordaje secuencial a los problemas, con un enfoque de carácter lineal y predecible. Aparentemente, este es el modelo que vemos en acción cuando se asume que por el tan solo hecho de construir un edificio moderno para una escuela periférica, se soluciona el tema del aprendizaje de los estudiantes y en definitiva la educación de los sectores más vulnerables.

De acuerdo a Morin, "El paradigma de simplificación -disyunción y reducción-es un paradigma que pone orden en el universo, y persigue al desorden, la simplicidad ve a lo uno y ve a lo múltiple" (2005:89); desde esta perspectiva este paradigma no es capaz de responder a los contextos en los que se sitúa hoy el sistema educativo, claramente -como lo demuestra la evidencia empírica- el problema de la educación no se resuelve con una inyección de recursos por sustantiva que esta sea, podemos cambiar el espacio físico, instalar tecnología de punta y levantar un nuevo currículo, no obstante, si actuamos desde la separación y la especialización sólo lograremos abordar parte del fenómeno, nunca el fenómeno en su multidimensionalidad y totalidad. 
Se requiere en consecuencia, un ejercicio sistemático de configuración, deconfiguración y reconfiguración que permita analizar el sistema escuela desde un paradigma recursivo, dialógico, hologramático, tal y como lo expone uno de los autores que más ha reflexionado sobre la concepción de complejidad: Edgar Morin.

El paradigma de complejidad provendrá del conjunto de nuevos conceptos, de nuevas visiones, de nuevos descubrimientos y de nuevas reflexiones que van a conectarse y reunirse, el principio de la complejidad, de alguna manera, se fundará sobre la predominancia de la conjunción compleja (Morin: 2005:I I0).

Por otro lado, y a objeto de intentar comprender el fenómeno educativo, es menester aproximarnos al sistema escuela desde una perspectiva fenomenológica (Rodríguez, Gil y García 1996), la que por cierto, no se reduce a "estudiar el fenómeno", sino más bien, a aproximarnos al "sentido del fenómeno", a su lenguaje, a su estructura, y no desde fuera, muy por el contrario, para poder sentirlo debemos ser parte del fenómeno, ser actores y co-creadores del mismo, nos olvidamos del objeto para convertirnos en sujeto y fundamentalmente un sujeto en relación que aspira a conocer la realidad o más bien la multidimensionalidad de la realidad, de acuerdo a lo expresado por Rozo: "con el término real se nombra aquello que se quiere conocer, lo real es el enigma, motivo de curiosidad que motiva el conocer humano" (2003:45).

En consecuencia, el paradigma racionalista es insuficiente para "comprender" el sistema escuela, puesto que este enfoque enseña a ver las cosas en una determinada dirección y al interior de un mundo ordenado, como si el universo fuese cerrado, lineal, determinado, pensamiento propio de la física clásica, el cual se derrumba con el advenimiento de una nueva ciencia:

La mecánica quántica cambia la comprensión de un mundo de objetos por la de un mundo configurado por partículas elementales, comprensión en la cual una partícula elemental no es un ente que existe independiente y escapa al análisis. En 
esencia es un conjunto de relaciones dirigido hacia otras cosas (Rozo, 2003: 3I).

Nuevamente la idea de incertidumbre, sistema, relacionalidad, complejidad, son los conceptos que emergen para enriquecer el fenómeno que pretendemos estudiar, al abrir las posibilidades en un bucle recursivo de afuera-adentro y de adentro-afuera, que se despierta como el árido desierto con las lluvias invernales, que viajan hacia el norte para deleitarnos con la maravillosa naturaleza del desierto florido en un juego mágico de formas, aromas y colores, y ese es quizás, el desafío para la escuela del tercer milenio.

\section{Concepción epistemológica}

Si a esta altura podemos concluir que sólo a través del paradigma de la complejidad es posible analizar el sistema educativo, ahora es momento de revisar los aspectos epistemológicos que subyacen en la dinámica relacional de la escuela, vale decir, cómo en la escuela operan los procesos del conocer, en el entendido que la educación actual privilegia una forma de concebir el conocimiento, de desarrollarlo y de gestionarlo, no obstante debería considerar lo expuesto por Vasilachis "la epistemología se interroga acerca de cómo la realidad puede ser conocida, acerca de la relación entre quien conoce y aquello que es conocido" (2006:46)

Como primera aproximación diremos que la escuela del siglo XXI se encuentra en un proceso de revisión y reflexión sobre su propia práctica, cuestión que es altamente valorada; no obstante, es posible visualizar todavía algunas estructuras y consecuentemente su organización, que aparentemente dan cuenta de una mirada más bien fragmentada del conocimiento; he aquí algunos ejemplos para visibilizar el fenómeno:

- Un currículo asignaturista que privilegia la separación de los saberes, y raramente genera la inter-multidisciplinariedad (Morin, 200I). 
- Una gestión curricular que discrimina algunas áreas del conocimiento, el ejemplo más evidente es la carga horaria de lenguaje y matemáticas, en relación a las horas de educación artística y educación física, por ejemplo.

- Conectado con lo anterior, la implementación de un sistema de evaluación nacional (SIMCE), que no recoge la muldimensionalidad del ser humano, sino más bien pretende medir la dimensión cognitiva de los estudiantes.

- Una práctica pedagógica aislada y descontextualizada, que genera más resistencias que emociones que favorezcan el aprendizaje.

- Un abordaje metodológico desde la descripción, la explicación y con fuerte énfasis en el contenido.

- Una relación profesor-alumno desde el educare, depositar, vaciar, lo que Paulo Freire denomina como educación bancaria (Freire, 2002).

Podríamos continuar enumerando situaciones que se desarrollan habitualmente en el contexto escolar y que solo viene a ratificar la manera como la escuela -en términos genéricos- concibe el acto de conocer. Aparentemente, la escuela ha entendido que la división y la separación son acciones absolutamente normales que no merecen un cuestionamiento e interpelación, por tanto, para poder "conocer" es menester fraccionar, puesto que al conocer las partes, podemos conocer el todo. Por otro lado, este mismo modelo promueve una relación sujeto-objeto a partir de la disociación, separando al sujeto del objeto, de tal manera, que la reflexión y la filosofía se ubican a un lado de la pizarra y la ciencia y la investigación objetiva se encuentran en la posición opuesta, sin posibilidad de vincularse y conectarse, cuestión planteada hace más de tres siglos por Galileo, Newton, Descartes y Bacon.

En este contexto, la propuesta es levantar un nuevo paradigma -quizás no tan nuevo, sino más bien el de siempre- que da cuenta de una epistemología concebida a partir de la unidad del conocimiento, en una concepción de carácter holístico y hologramático en el que el todo está presente en las partes y las partes presentes en el todo, 
generando una serie de relaciones de tan alta magnitud que solo es posible aproximarnos a ella a través del paradigma de la complejidad, el que nos permite captar lo que está "tejido conjuntamente", en palabras de Morin "se trata de comprender un pensamiento que separa y que reduce junto con un pensamiento que distingue y religa" (200I:45).

Consecuentemente, la tarea de la escuela -al parecer- debiera ser transitar desde un conocimiento simplista hacia un conocimiento complejo, en el cual no existe separación, sino más bien, el conocimiento se entiende como un todo que se teje, enhebra, imbrica y se relaciona desde múltiples posibilidades, por tanto, no podría existir la clásica separación entre teoría - práctica, razón - emoción, ciencia - filosofía, puesto que estas acciones son inseparables e indisociables para poder precisamente "conocer" el fenómeno que se investiga. De acuerdo a lo planteado por Rozo, "Morin redimensiona el problema del conocimiento y las formas de su elaboración. Ve en la filosofía y en la ciencia dos perspectivas complementarias y necesarias, propone una reorganización epistemológica” (2003:44), proponiendo precisamente volver a la unidad del conocimiento, tal y como lo experimentaban los pueblos originarios de nuestra América precolombina en sus diversos, complejos y mágicos actos de conocer y posteriormente el año 1600 el gran pensador Giordano Bruno, quien pagó en la hoguera de la inquisición la audacia de concebir la infinitud del universo (Vit, 2010).

¿Es posible que la escuela del siglo XXI de-configure su concepción epistemológica? Si la respuesta es positiva surge la segunda cuestión: ¿podrá plantear la escuela una re-configuración del conocimiento desde una perspectiva compleja? Desde la intuición, nos inclinamos por señalar que para lograr lo anterior deben darse algunas señales como las siguientes:

- Transitar desde una concepción que privilegia el desarrollo económico y la producción, a una concepción que favorezca y posibilite el desarrollo humano (Savater, en Carrasco, Negrón y Astorga, 2006). 
- Romper con los clásicos dualismos en los que se funda la práctica pedagógica tradicional, profesor - alumno, razón emoción, cuerpo - mente, sujeto - objeto, ciencia - filosofía, comprendiendo que el niño y la niña son una unidad y una multiplicidad a la vez (Damasio, 2006).

- Desarrollar con los estudiantes una autonomía relacional, vale decir, hacer lo que otorga sentido, en relación con los otros, considerando la historicidad y el contexto de la persona humana.

- Levantar una propuesta curricular que dé cuenta de una visión inter-multi-transdisciplinaria, que posibilite la emergencia de una epistemología compleja, en el marco de lo expuesto por Morin (1994).

La implementación de esta manera de mirar el fenómeno, debiera conducir a lo planteado en párrafos anteriores en orden a reconfiguar el sistema escuela, adecuando su paradigma epistemológico a un tiempo en el que la forma de construir y desarrollar el conocimiento es lo central del proceso educativo, tal y como lo expresa Morin: "por consiguiente, el desarrollo de la aptitud para contextualizar y totalizar los saberes se convierte en un imperativo de la educación” (2002:27).

\section{Concepción sistémica de la escuela}

La escuela forma parte del sistema educativo, el que se encuentra incorporado al sistema social, constituyendo por tanto, un subsistema del sistema social. La escuela posee características que le son propias como su organización interna, su cultura, su relación con la comunidad, su forma de desarrollar el aprendizaje, en definitiva posee una particular concepción de hombre y de mundo, que ha construido gracias a las relaciones con el medio y a las propias interacciones de sus elementos constitutivos, que la convierten en un sistema único, rico y complejo.

El concepto de "sistema educativo" es frecuentemente utilizado por quienes abordan el estudio y la investigación en el ámbito educacional; 
la pregunta que surge sin embargo es, ¿cuál es la concepción de sistema a la cual se hace alusión?, ¿qué entendemos por sistema?, ¿en qué paradigma epistemológico se ancla esta concepción? Intentaremos a continuación desarrollar algunos elementos que desde nuestra mirada permitirán comprender y acercarse a una concepción sistémica de la escuela desde el paradigma de la complejidad.

En primer término diremos que el paradigma clásico de disyunción, determinismo, orden, causalidad lineal y de objetividad ha sido el gran referente para generar conocimiento, incluso en las propias ciencias humanas, al adoptar éstas el modelo investigativo de las ciencias naturales. Lo anterior ha generado -de acuerdo a lo expuesto en párrafos anteriores- una concepción de sistema que responde a este paradigma, el que se expresa y devela en frases como las siguientes: "para conocer el todo, sólo hace falta conocer las partes", "el todo no es más que la suma de las partes"; por otro lado, se entiende que los elementos constitutivos del sistema son "ingreso - proceso - salida”, y serían suficientes para comprender el sistema. Esta lógica de linealidad y secuencialidad favorece la fragmentación que se observa en el sistema escolar, puesto que es el soporte teórico de una práctica aislada y poco reflexiva que se funda a partir de la simplicidad.

A objeto de precisar la propuesta recurriremos a Rozo, quien señala:

La palabra sistema viene del griego synistanai, y contiene nociones que significan reunir, juntar, colocar juntos. En la actualidad el concepto de sistema hace referencia a una unidad, un todo integrado, un conjunto, cuyas propiedades y características emergen del juego de relaciones y conexiones entre los elementos que lo configuran y del todo con el entorno en el cual se halla inscrito (Rozo, 2003:5I).

Ahora bien, desde este enfoque ¿cuáles serían los elementos que configuran un sistema? y más específicamente, cómo se constituye y acciona el sistema escuela: 
- Elementos autónomos: el sistema estaría compuesto por elementos con "vida" propia, estos elementos son similares pero diferentes entre sí, vale decir, en la escuela los alumnos, el profesorado, los directivos, el currículo, los padres, la comunidad, los espacios de aprendizaje, la didáctica, y otros, poseen características propias que los señalan y les otorgan identidad. Por otro lado, es necesario comprender que su relacionalidad no es sumatoria, sino más bien, probabilística y altamente compleja.

- Interrelación e interconexión: el sistema se constituye a partir de las relaciones y conexiones entre los elementos y el todo del cual forman parte, en consecuencia, para nuestro análisis a nivel de escuela, los elementos (alumnos, profesores, familia...), juegan a relacionarse entre ellos y con la escuela -entendida esta como totalidad-, emergiendo entonces una relacionalidad azarosa, puesto que no es posible predecir su desarrollo desde la causalidad y desde la sumatoria.

- Reorganización del sistema: el sistema se configura, de-configura y re-configura permanentemente a partir de la red de relaciones -que se sitúan en redes mayores- que se dan en su medio y con su entorno. Desde esta perspectiva la escuela se organiza y reorganiza permanentemente a través de la interretroacción del todo sobre las partes y viceversa. Por tanto, no es posible estudiar sólo a los alumnos sin sus relaciones y su contexto.

- Posibilidades y probabilidades: nada es predecible, nada es una certeza, todo es sólo una posibilidad que emerge desde la relacionalidad sistémica. En consecuencia, la escuela debe optar, tomar decisiones frente al abanico de oportunidades que se generan al interior de sus aulas, patios, oficinas... y con su entorno.

De acuerdo a lo expresado precedentemente, este enfoque sistémico propuesto para abordar el estudio y el análisis del sistema escuela, rompe con la mirada tradicional que jibariza y reduce el fenómeno a una sumatoria de eventos y posibilidades lineales; muy por el contrario, lo que se pretende desde esta perspectiva es conectarlo fuertemente con el paradigma de la complejidad, entendiendo a la escuela como una organización que en palabras de Morin sería: 
La organización une, transforma, produce, mantiene. Une, transforma los elementos en un sistema, produce y mantiene este sistema... liga los elementos entre sí, los elementos en una totalidad, los elementos a la totalidad, la totalidad a los elementos, es decir, une entre sí todas las uniones y constituye la unión de las uniones... la organización da forma en el espacio y en el tiempo a una realización nueva: la unidad compleja o sistema (Rozo, 2003:66).

Precisamente este es el desafio que se nos presenta, comprender a la escuela desde una concepción sistémica, eminentemente compleja que se autoorganiza permanentemente en su relacionalidad consigo misma y con el entorno.

\section{La escuela ¿posibilidades de mudanza?}

¿Qué ha llevado a que en la actualidad el concepto de cambio haya transversalizado tan fuertemente al fenómeno educativo? En la literatura especializada, encontramos referencias a "cambios hacia la calidad", "cambios en la formas de enseñar", "cambio en el currículo", "cambio en los profesores", "cambio en las prácticas pedagógicas" o incluso, "reformas en los sistemas educativos en su conjunto” como señales claras de procesos de cambio.

A simple vista pareciera que todo debe mudar y cambiar. Las preguntas, entonces, son ¿hacia dónde?, ¿qué significa cambio en el contexto educativo?, ¿se requiere de verdad el cambio? Intentaremos algunas aproximaciones al suceso, en el bien entendido que quizás jamás podamos atrapar el fenómeno, sólo aproximarnos primariamente a su comprensión.

En primer término, el concepto mudanza, de acuerdo a lo expuesto por Feitosa, es "una generosidad de la vida, es un principio de libertad y desenvolvimiento" (2006:23), en consecuencia, emerge como una posibilidad, como una problemática que no está dada, sino más bien, debe ser construida a la luz del paradigma de la complejidad, 
luego señala que "toda la epistemología actual habla de cambio, de la necesidad de mudanza, de la inevitabilidad del cambio” (ibídem: 24). Por consiguiente, cuando hablamos de educación y mudanza el tema de fondo es cómo se gesta y desarrolla el conocimiento precisamente en el lugar que ha privilegiado la sociedad para cultivar el saber. Por su parte Murillo (2003) explica el concepto de cambio diciendo que conlleva un carácter complejo y sistemático de actuación que va más allá de un cúmulo de experiencias aisladas y de su mera ordenación administrativa; significa abogar por una visión de la innovación suficientemente compleja y rica.

En estas enunciaciones encontramos ciertas características que permiten identificar cuándo estamos frente a un proceso de mudanzacambio. El carácter de sistemático -prolongado en el tiempoque es reconocido por los integrantes del sistema, que puede ser analizado, gestionado, evaluado y fundamentalmente que comprende relaciones complejas al interior del ser humano, serían los elementos que lo conforman y que en definitiva hacen la diferencia entre una innovación puntual y un proceso de cambio que es capaz de impactar positivamente al sistema educativo.

Cuando nos preguntamos hacia dónde se dirige el cambio, pareciera ser que el consenso es la posibilidad de mejora del sistema educativo, mejora asociada a calidad. Partimos entonces de dos premisas que se encuentran en la base de las innovaciones propuestas, la primera es que el sistema educativo tiene la capacidad de mejora, es decir, que sus procesos son perfectibles, y la segunda es que requiere de ciertas modificaciones en su estructura, puesto que lo que hoy existe no se ajusta a la transformación científica, tecnológica y sociocultural de la sociedad del conocimiento y la información. Una sociedad que comprende que el sistema educativo es gravitante para alcanzar a las nuevas generaciones, de tal modo, de levantar paradigmas epistemológicos que llevan implícitas nuevas formas de construir y gestionar el conocimiento. 


\section{6. ¿Y si la calidad en la escuela fuese una emergencia sistémica? (recreando a Boisier 2003)}

Durante los últimos días, el debate en educación se ha centrado en torno a los resultados de la aplicación del SIMCE el año 2010 y a los anuncios en materia de educación expuestos por el señor ministro de educación. Ambos temas están cruzados transversalmente por el concepto calidad, en el primer ámbito, asociado a aprendizaje, y en el segundo, vinculado con la gestión de las escuelas y liceos. Sin lugar a dudas el concepto calidad educativa posee una gran dispersión semántica, por lo que resulta difícil una definición precisa que contemple el fenómeno en su conjunto; no obstante, para este ejercicio de reflexión entenderemos por calidad en el sistema escuela como la respuesta oportuna y éticamente responsable frente a lo que espera la sociedad de la acción educativa y más específicamente, la calidad estaría referida a que los niños y niñas sean formados como personas con una estructura valórica coherente y con aprendizajes que respondan a las competencias requeridas en la sociedad del siglo XXI.

En este contexto, la gran discusión en las pasadas dos décadas ha sido precisamente conseguir la denominada "calidad educativa", lo que aparentemente no se ha conseguido puesto que desde los propios agentes involucrados, alumnos y profesores -recordemos las manifestaciones de mayo del año 2006-, hasta la clase política, no están conformes con lo que hoy está generando el sistema escolar. De acuerdo al discurso planteado existen problemas de desigualdad, discriminación, recurso humano insuficiente y sin la preparación requerida, autonomía y gestión de los establecimientos, currículos descontextualizados, prácticas pedagógicas lineales, escasa participación de la comunidad y vinculación con el medio, y lo más preocupante, un nivel de logro en los aprendizajes de los estudiantes más bien bajo. En fin, se plantean una serie de argumentos para sostener que el sistema educativo actual no está respondiendo a las exigencias del medio, por tanto, su calidad es insuficiente. 
En relación al concepto de emergencia, podemos aproximarnos señalando que a partir de dos cosas distintas aparece la novedad, vale decir, una tercera opción diferente a las anteriores, tal y como lo expresa Varela. "Este paso desde normas locales a una coherencia global es el corazón de lo que solía llamarse autoorganización durante los años fundacionales. Hoy en día, se prefiere hablar ya sea de propiedades emergentes o globales" (2010: 193). En consecuencia, situados en el dominio de la escuela, podríamos afirmar que en la relacionalidad del sistema emergen cualidades diferentes. Desde esta mirada el enfoque pasa a ser eminentemente analógico, puesto que posibilita hacer nuevas distinciones, he aquí entonces la riqueza de esta nueva mirada del fenómeno educativo en términos de la multidimensionalidad de posibilidades que otorga.

Volviendo al concepto de calidad, la impresión es que generalmente se aborda desde una mirada más bien reduccionista y mecanicista, toda vez que para mejorar la "calidad" de la escuela se implementan acciones como:

- Mejora en la infraestructura

- Perfeccionamiento de los profesores

- Desarrollo de las TIC

- Creación y desarrollo de un proyecto educativo

- Convenios y vínculos con la comunidad

- Entrega de textos escolares

- Aumento del tiempo escolar JEC

- Reforma curricular

- Becas de alimentación

- Práctica pedagógica de carácter constructivista

- Constitución de consejos escolares

- Ley SEP

- Etc.

Es posible continuar explicitando el sinnúmero de acciones que se desarrollan en la escuela a objeto de elevar su "calidad", no obstante, 
si se continua abordando el fenómeno desde la sumatoria de acciones, desde lo discreto, desde lo lineal, evidentemente los resultados serán en esta misma lógica. En consecuencia, no podría haber calidad educativa desde un paradigma que se fundamenta en el método analítico, marcadamente cuantitativo y cuya concepción de sistema responde a las categorías "entrada - proceso - salida”, en definitiva, un paradigma que está anclado aún en la disyunción cartesiana.

Desde una aproximación fenomenológica (Maturana, 2005; Echeverría, 2009) visualizamos la calidad de la educación como una cualidad emergente que se despliega al interior-exterior del sistema bajo un paradigma eminentemente complejo, multidimensional, cualitativo y holístico. A lo anteriormente expuesto y en relación al concepto de emergencia, podemos complementarlo con lo planteado por Boisier: "las propiedades emergentes se definen como fenómenos culturales y sociales que emergen de las interacciones e intercambios entre los miembros de un sistema social" (2003:5I). En este contexto, la propuesta es re-mirar el fenómeno bajo las siguientes premisas:

- Enfoque sistémico: el que se traduce en contextualizar la escuela, estableciendo la naturaleza de sus relaciones y reconociendo sus conexiones, abriendo un espacio para la relacionalidad desde la unidad a la totalidad y de la totalidad a la unidad.

- Pensamiento complejo: aplicado en el sistema escuela como el privilegio de la unión, la conjunción, diversidad, azar, incertidumbre, que utiliza el principio dialógico de ordendesorden-organización, un pensamiento que integra la relación entre las partes y el todo de manera recursiva y que nos permite transitar del archipiélago al continente.

- Concepción epistemológica: la escuela es el lugar en que precisamente se crea y desarrolla el conocimiento, vale decir, es el lugar elegido como centro de re-creación del saber de la humanidad, por tanto, las preguntas ¿qué es conocer? y ¿cómo se conoce?, deben ser parte del diálogo y la reflexión al interior de ella. 
A partir de las tres condiciones precedentemente señaladas, sería posible hablar de una emergencia sistémica, en el bien entendido que no es la sumatoria de acciones lo que genera la calidad en la escuela, sino más bien, una simultaneidad que la hace surgir, que la hace emerger, vale decir, no es posible pensar en calidad educativa en el sistema escuela si no es bajo el paradigma del enfoque sistémico, el pensamiento complejo y una nueva concepción epistemológica, como una tríada mágica que se despliega y toma forma a partir de la relacionalidad, en una comunicación permanente del sistema consigo mismo y con el entorno.

\section{A modo de conclusión}

En definitiva, en este ejercicio reflexivo, que me he permitido traducir en este documento, emerge también mi convicción profunda de que las posibilidades del sistema escuela -en este entorno de vertiginosos y constantes cambios- se encuentran precisamente al interior de ella, puesto que en la escuela están todas las posibilidades para hacer emergen una cualidad diferente, cual es, precisamente, la ansiada "calidad educativa", no obstante, para conseguirlo es menester reconfigurar nuestra propia práctica y principalmente incorporar los conceptos y enfoques de vanguardia en torno a la creación y desarrollo del conocimiento. En este contexto, la tarea del sistema en su conjunto sería precisamente revisar, analizar y reflexionar acerca de los paradigmas y enfoques planteados, a objeto de incorporarlos en su dinámica operacional, en el entendido que "el futuro será una puerta... que no sabe cerrarse" (Benedetti 200I).

\section{Bibliografía}

Benedetti, M. (200I). El mundo que respiro. Buenos Aires. Planeta.

Boisier, S. (2003). “ ¿Y si el desarrollo fuese una emergencia sistémica?”. En Revista Reforma y Democracia. N 27. Caracas.

Carrasco, E.; Negrón, B.; Astorga, A. (2006). Sentidos de la educación y la cultura: cultivar la humanidad. Santiago. Lom. 
Damasio, A. (2006). El error de Descartes, $4^{\text {ta. }}$ Impresión. Barcelona. Crítica.

Echeverría, R. (2009). Escritos sobre aprendizaje, recopilación. Santiago. JC Sáez.

Feitosa, A.; Kolyniak, C. y Rath, H. (2006). Mudanzas: horizontes desde la motricidad. Popayán, Universidad del Cauca.

Freire, P. (2002). Pedagogía de la esperanza, un reencuentro con la pedagogía del oprimido. Buenos Aires. Siglo XXI

Maturana, H.; Porksen, B. (2005). Del Ser al Hacer. Los orígenes de la biología del conocer. Santiago. JC Sáez.

Morin, E. (I994). El método III. El conocimiento del conocimiento. Madrid. Cátedra.

Morin, E. (200I). Los siete saberes necesarios para la educación del futuro. Barcelona, Paidós.

Morin, E. (2005). Introducción al pensamiento complejo. Octava reimpresión. Barcelona. Gedisa.

Murillo, P. (2003). "Los procesos de cambio en la sociedad actual: resistencias y nuevos retos". Conferencia presentada en las IV Jornadas de Educación de la Salud "Innovación y Cambio en la Docencia". Universidad de Chile, Santiago, mayo de 2003.

Rodríguez, G.; Gil, J. y García, E. (I996). Metodología de la Investigación Cualitativa. Málaga. Ediciones Aljibe.

Rozo, J. (2003). Sistémica y pensamiento complejo. Bogotá. Biogénesis.

Varela, F. (2010). El fenómeno de la vida. Santiago. JC Sáez.

Vasilich, I. (2006). Estrategias de Investigación Cualitativa. Barcelona. Gedisa.

Vit, L. (2010). Giordano Bruno, forastero en el universo. Bogotá. Debolsillo. 